\title{
Stokes Problem for the Generalized Navier-Stokes Equations
}

\author{
Andrei Bourchtein, Ludmila Bourchtein \\ FAPERGS Science Foundation/Pelotas State University, Department of Mathematics \\ Campus Universitario da UFPel, 96010-900, Brazil \\ E-mail: burstein@conex.com.br
}

\begin{abstract}
The generalized Navier-Stokes equations for incompressible viscous flows through isotropic granular porous medium are considered. First Stokes problem is solved applying Laplace transform with respect to time variable and evaluating the inverse transform integrals by the residue calculus. The derived analytical solution includes the classic one as a particular case, that is, it can be obtained from the generalized solution by putting the porosity parameter equal to 1 . The use of the derived exact solutions for benchmarking purposes is described.
\end{abstract}

\section{Introduction}

Due to nonlinearity of the Navier-Stokes equations only a small number of exact solutions have been found. The most recent reviews of analytical solutions of the Navier-Stokes equations and its classification were given by Wang [7,8]. These solutions are important because they represent some fundamental fluid flows and serve for checking the accuracy of approximate methods, in particular, numerical schemes. We consider one model of laminar flows through granular porous medium which can be represented in the form of the generalized Navier-Stokes equations. It is useful to generalize some known analytical solutions of the Navier-Stokes equations to the case of the considered model. Couette-Poiseuille, Hagen-Poiseuille and Beltrami generalized solutions were obtained by Bourchtein et al. [1]. Here we derive the Stokes generalized solution and show its possible application for benchmarking.

\section{Differential problem}

The generalized Navier-Stokes equations applied to the description of incompressible viscous laminar flows through a rigid isotropic granular porous medium have been developed by DuPlessis and Masliyah [3]. The advantages of their model are its applicability to granular porous media over the entire porosity range and simple adaptability to numerical simulations. These equations have the following form: 


$$
\rho \mathbf{V}_{t}+\rho(\mathbf{V} \cdot \nabla) \frac{\mathbf{V}}{n}=-n \nabla p+\mu \nabla^{2} \mathbf{V}-\mu F \mathbf{V}-n \rho \mathbf{g}, \nabla \cdot \mathbf{V}=0
$$

Here, the common denotations are used for fluid and porous medium characteristics: $\mathbf{V}$ is a fluid velocity vector, $p$ is a pressure; $\mathbf{g}$ is the gravitational force. The fluid is specified by definition of density $\rho$ and dynamic viscosity $\mu$. The characteristics of porous medium are porosity $n$ and porosity function $F$. Porosity $n$ is defined as a ratio of volume of the void space to the bulk volume of a porous medium and it changes from zero to one $(n \in(0,1])$. Function $F$ represents an additional drag force, which describes influence of porous medium on flow. This function depends on porosity $n$ only, it is continuous, decreasing and positive on interval $(0,1] . F$ becomes infinite as $n$ approaches zero and its limit is equal to 0 as $n$ approaches 1 . Comparing this system with the usual incompressible Navier-Stokes equations, one can see that the latter is the particular case of model (1) when $n=1$.

Considering the primitive system in $4 \mathrm{D}$ domain $[0, T] \times \bar{\Omega}$, where $\bar{\Omega}=\Omega+\partial \Omega$ is a 3D space bounded domain with boundary $\Gamma=\partial \Omega$, we have to specify the initial and boundary conditions to define the unique solution:

$$
\mathbf{V}=\mathbf{V}_{0} \text { on } \bar{\Omega} \text { at } t=0
$$

(initial condition, $\mathbf{V}_{0}$ is the given function of spatial variables),

$$
\mathbf{V}=\mathbf{V}_{\Gamma} \text { on } \Gamma=\partial \Omega \text { for all } t \in[0, T]
$$

(no-slip boundary condition, $\mathbf{V}_{\Gamma}$ is the given function of the time variable and two spatial variables). These conditions have to subject to some constraints specified in $[2,5]$. We assume that the last constraints are satisfied by the appropriate choice of initial and boundary conditions, so we will not mention these conditions anymore.

\section{Solution of Stokes problem}

We consider the problem of unsteady flow of a semi-infinite fluid, which is caused by a plate moving in its own plane. There are two independent variables in this problem: the time $t$ and the spatial variable $y$ (a figure can be found in [9]). Using the simplification conditions $v \equiv Q, w \equiv 0$, primitive system (1) can be reduced to the following initial boundary value problem involving velocity component $u$ :

$$
u_{t}=u_{y y}-\mathcal{F} u, u(t, 0)=A, u(t,+\infty)=0, u(0, y)=0 .
$$

Here $v=\mu / \rho$ is the kinematic viscosity. In the classic case (when $F=0$ ) the solution of this problem can be found by the similarity method, defining the only independent variable of the problem and reducing the primitive bivariate partial differential equation to an ordinary one $[7,9]$. Unfortunately, an introduction of 
additional porous drag force eliminates this way of derivation of solution, because there are two dimensionless combinations of primitive variables in this case. So we have been forced to choose another method. Due to linearity and parabolicity of the $u$-component equation (4) the Laplace transform with respect to variable $t$

$$
U(s, y)=L[u(t, y)] \equiv \int_{0}^{\infty} u(t, y) e^{-s t} d t
$$

is suitable to be applied. This transform reduces (4) to an ordinary differential problem:

$$
U_{y y}=\frac{s}{v} U+F U, U(s, 0)=\frac{A}{s}, U(s,+\infty)=0 .
$$

A solution of (6) is

$$
U=\frac{A}{s} e^{-\sqrt{\frac{s}{v}+F y}} .
$$

Applying inverse transform we obtain solution of (4) in the form

$$
u(t, y)=L^{-1}[U(s, y)] \equiv \frac{1}{2 \pi i} \int_{c-i \infty}^{c+i \infty} U(s, y) e^{s t} d s=\frac{1}{2 \pi} \int_{c-i \infty}^{c+i \infty} \frac{A}{s} e^{-\sqrt{\frac{s}{v}+F y}} e^{s t} d s .
$$

To evaluate the integral on the right hand side of (8) we change variable $s$ and parameter $y$ :

$$
z=s+\sqrt{F}, d=\frac{y}{v}
$$

and we get

$$
\frac{1}{2 \pi} \int_{c-i \infty}^{c+\infty \infty} \frac{A}{s} e^{-\sqrt{\frac{\rho}{\mu} s+F} y} e^{s t} d s=\frac{1}{2 \pi \dot{d}} \int_{c+v F-i \infty}^{c+v^{F}+\infty \infty} \frac{A}{z-\sqrt{F}} e^{-\sqrt{z} d} e^{z t} e^{-v F t} d z=\frac{A e^{-v F t}}{2 \pi} \int_{q^{-i \infty \infty}}^{\mathrm{q}+i \infty} g(z) d z,
$$

where

$$
g(z)=\frac{1}{z-\sqrt{F}} e^{-\sqrt{z} d} e^{z t}, c_{1}=c+\mathcal{W} .
$$

Function $\sqrt{z}$ can be made a single-valued regular function of $z$, if a cut along the negative real axis is applied to the $z$-plane. In this case, function $e^{-\sqrt{z} d}$ will also be a single-valued regular function. We choose the branch of $\sqrt{z}$ for which $\sqrt{1}=1$.

Let us consider a closed contour $C=C_{R} \cup C_{+} \cup C_{-} \cup C_{r} \cup C_{l}$ shown in Fig.1, where $C_{R}$ is the part of the circular arc with radius $R>c_{1}$ lying on the left of line $\operatorname{Re} z=c_{1}$, $C_{r}$ is a circular arc with radius $r<c_{1}, C_{+}$and $C_{-}$are the parts of upper and lower edges of the cut along the negative real axis, connecting the circular arcs with radius $R$ and $r, C_{l}$ is the part of line $\operatorname{Re} z=c_{1}$ inside the circle with radius $R$. 


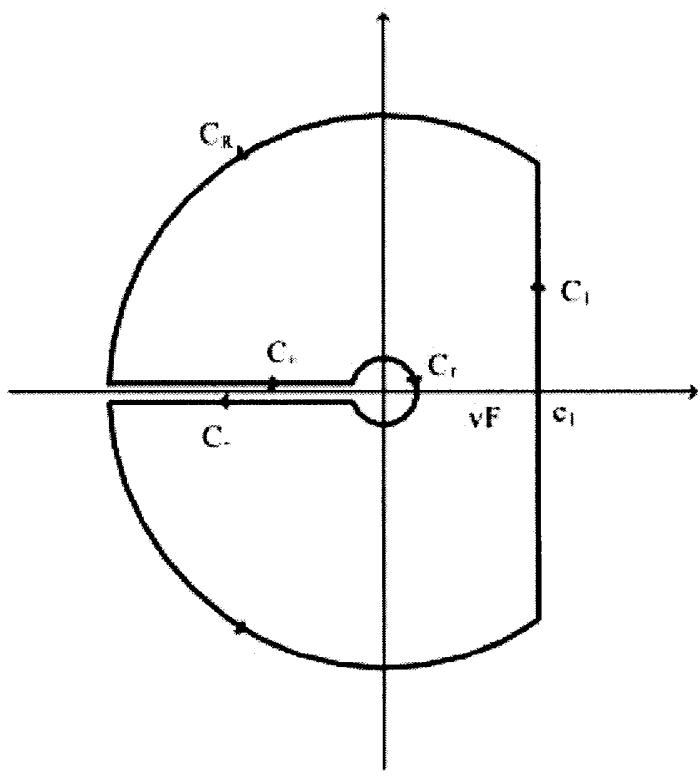

Figure 1. Closed contour $C$ and its partition $C=C_{R} \cup C_{+} \cup C_{-} \cup C_{r} \cup C_{l}$.

The chosen branch of the function $g(z)$ is regular in the simply-connected domain $D$ bounded by closed contour $C$ except at a point $z=\sqrt{F}$ where it has a simple pole. Therefore, by the residue theorem,

$$
\int_{C} g(z) d z=2 \pi \dot{\pi} \cdot \operatorname{res}_{z=V F} g(z)=\left.2 \pi \dot{\pi} \cdot e^{-\sqrt{z} d} e^{z t}\right|_{z=F F}=e^{-\sqrt{V F} d} e^{v F t}
$$

On the other hand, we can represent the integral along $C$ as the sum of the integrals along respective parts of contour $C$ (see Fig.1):

$$
\int_{C} g(z) d z=\int_{C_{R}}+\int_{C_{+}}+\int_{C_{-}}+\int_{C_{r}}+\int_{C_{l}} g(z) d z
$$

Formulas (12) and (13) are true for any radius $R$ and $r$ such that $R>c_{1}>r$.

Let us evaluate each integral in (13) separately, as $R$ approaches $\infty$ and $r$ approaches 0:

1) The chosen single-valued branch of function $\sqrt{z}$ has value $\sqrt{R} e^{i \varphi / 2}$ at the points of $C_{R}$. Then

$$
\left|\frac{e^{-\sqrt{z} d}}{z-\sqrt{F}}\right|_{C_{R}}=\left|\frac{e^{-\sqrt{R} d(\cos \varphi / 2+\sin \varphi / 2)}}{\operatorname{Re}^{i \varphi}-\sqrt{F}}\right| \leq \frac{e^{-\sqrt{R} d \cos \varphi / 2}}{R-\sqrt{F}} \underset{R \rightarrow \infty}{\longrightarrow} 0
$$


because

$$
-\frac{\pi}{2} \leq \frac{\varphi}{2} \leq \frac{\pi}{2}, \cos \frac{\varphi}{2} \geq 0, d>0 .
$$

Therefore, by Jordan's lemma [6],

$$
\int_{C_{R}} g(z) d z \underset{R \rightarrow \infty}{\longrightarrow} 0 \text {. }
$$

2,3) Now we evaluate together the second and third integrals in (13)

$$
\begin{gathered}
\int_{C_{+}} g(z) d z+\int_{C_{-}} g(z) d z=\int_{R-a-\sqrt{F}}^{r} \frac{1}{-i \sqrt{a} d} e^{-a t}(-d a)+\int_{r}^{R} \frac{1}{-a-\sqrt{F}} e^{i \sqrt{a} d} e^{-a t}(-d a)= \\
=2 i \int_{r}^{R} \frac{\sin \sqrt{a} d}{a+\sqrt{F}} e^{-a t} d a \underset{R \rightarrow \infty, r \rightarrow 0}{\longrightarrow} 2 i \int_{0}^{+\infty} \frac{\sin \sqrt{a} d}{a+\sqrt{ }} e^{-a t} d a .
\end{gathered}
$$

Making the substitution $a=x^{2}$, we obtain

$$
\int_{0}^{+\infty} \frac{\sin \sqrt{a} d}{a+v F} e^{-a t} d a=2 \int_{0}^{+\infty} \frac{\sin x d}{x^{2}+v F} e^{-t x^{2}} x d x
$$

The last integral can be found in [4]:

$$
\left.2 \int_{0}^{+\infty} \frac{\sin x d}{x^{2}+\sqrt{F}} e^{-t t^{2}} x d x=-\frac{\pi}{2} e^{t F}\left[2 \sin \sqrt{V^{F}} d+e^{-\sqrt{F^{F}} d} \Phi \sqrt{t \sqrt{F}}-\frac{d}{2 \sqrt{t}}\right)-e^{\sqrt{V^{F}} d}\left(\sqrt{t \sqrt{F}}+\frac{d}{2 \sqrt{t}}\right)\right]
$$

where

$$
\Phi(x)=\frac{2}{\sqrt{\pi}} \int^{x} e^{-t^{2}} d t
$$

is the probability integral.

4) Since the chosen branch of $g(z)$ is regular in neighborhood of the point $z=0$ cutting along negative real axis, there exists a number $M$ such that $|g(z)| \leq M$ for all $z$ in this neighborhood. Therefore

$$
\left|\int_{C_{r}} g(z) d z\right| \leq \int_{C_{r}}|g(z)| d z \mid \leq M \cdot 2 \pi \underset{r \rightarrow 0}{\longrightarrow} 0
$$

5) It is evident that

$$
\int_{C_{l}} g(z) d z \underset{R \rightarrow \infty}{\longrightarrow} \int_{q-i \infty}^{q^{+i \infty}} g(z) d z
$$


Finally, putting the right hand sides of (12) and (13) to be equal, calculating limits as $R$ approaches $\infty$ and $r$ approaches 0 and using the results (16), (19), (21), (22), we obtain

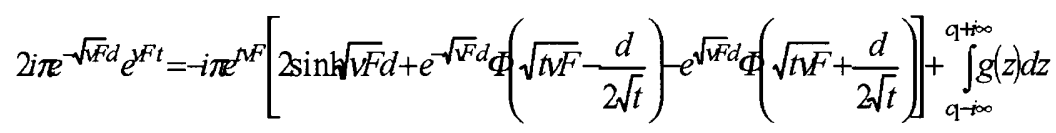

Therefore, returning to (8), we can express the solution of (4) in the following form

$$
\left.u(t, y)=\frac{A e^{y F t}}{2 \pi}\left\{2 \pi e^{-x \sqrt{F v}} e^{F t}+i \pi e^{\sqrt{F}}\left[2 \sinh \sqrt{F v}+e^{-y \sqrt{F v}} \Phi\left(\sqrt{t F}-\frac{y}{2 \sqrt{t v}}\right)-e^{y \sqrt{F / v}} \phi \sqrt{t \sqrt{F}}+\frac{y}{2 \sqrt{t v}}\right)\right]\right\}
$$

or, simplifying,

$$
u(t, y)=A \cosh y \sqrt{F / v}+\frac{A}{2}\left[e^{-y \sqrt{F / v}} \Phi\left(\sqrt{t v F}-\frac{y}{2 \sqrt{t v}}\right)-e^{y \sqrt{F / v}} \Phi\left(\sqrt{t v^{F}}+\frac{y}{2 \sqrt{t v}}\right)\right] .
$$

It is evident that the initial condition is satisfied in limit form as $t$ approaches 0 because

$$
\Phi\left(\sqrt{t v F}-\frac{y}{2 \sqrt{t v}}\right) \underset{t \rightarrow+0}{\longrightarrow}-1, \Phi\left(\sqrt{t v F}+\frac{y}{2 \sqrt{t v}}\right) \underset{t \rightarrow+0}{\longrightarrow} 1 .
$$

To transform this generalized solution to classic one it is sufficient to set $F=0(n=1)$.

There are detailed tables and different programs for calculating the probability integral values, so the derived solution can be evaluated with any order of precision.

The application of this solution to benchmarking is simple. It is sufficient to introduce bounded domain with respective boundary conditions. For example, considering equation (1) in rectangular parallelepiped and using Cartesian coordinates, we can complete it by initial conditions (2) and boundary conditions ( 3 ) specified as follows:

$$
\begin{gathered}
v_{t=0}=v_{0}=0, w_{t=0}=w_{0}=0, u_{t=0}=u_{0}=0 ; \\
v_{\Gamma}=0, w_{\Gamma}=0, u_{z=0}=u_{z=c}=0, u_{x=0}=u_{x=a}=u(t, y), u_{y=0}=A, u_{y=b}=u(t, b) .
\end{gathered}
$$

\section{Conclusion}

First Stokes problem for generalized Navier-Stokes equations is considered. Laplace transform is applied to reduce this problem to the set of ordinary differential problems which permit simple solutions. Inverse transform is calculated using some results of the theory of complex variable functions. A possible application of obtained exact solution to benchmarking is discussed. 


\section{Acknowledgments}

We are grateful to the Brazilian foundation FAPERGS which supported this work by a grant $01 / 60053.9$.

\section{References}

1. Bourchtein, A., Bourchtein, L., Lukaszczyk, J.P.: Numerical simulation of incompressible flows through granular porous media. Applied Numerical Mathematics 40 (2002) 291-306

2. Constantin, P., and Foias, C. Navier-Stokes equations. University of Chicago Press, Chicago (1989)

3. DuPlessis, P.J., and Masliyah, J.H.: Flow through isotropic granular porous media. Transport in Porous Media, 6 (1991) 207-221

4. Gradshteyn, I.S., Ryzhik, I.M.: Tables of integrals, series and products. Academic Press, Boston (1994)

5. Gresho, P.M.: Incompressible fluid dynamics: some fundamental formulation issues. Annual Reviews of Fluid Mechanics, 23 (1991) 413-453

6. Shilov, G.E.: Elementary real and complex analysis. Dover Publications, New York (1996)

7. Wang, C.Y.: Exact solutions of the unsteady Navier-Stokes equations. Applied Mechanics Review, 42 (1989) 269-282

8. Wang, C.Y.: Exact solutions of the steady-state Navier-Stokes equations. Annual Reviews of Fluid Mechanics, 23 (1991) 159-177

9. Yih, C.-S.: Fluid mechanics. McGraw-Hill, New York (1977) 